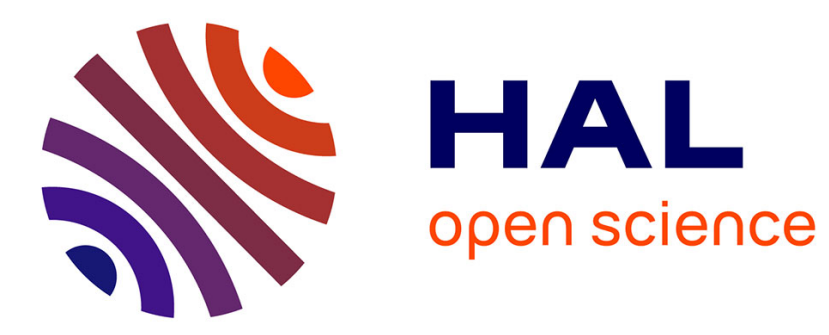

\title{
Orbital superlattice in the degenerate Hubbard model
}

\author{
M. Cyrot, C. Lyon-Caen
}

\section{To cite this version:}

M. Cyrot, C. Lyon-Caen. Orbital superlattice in the degenerate Hubbard model. Journal de Physique, 1975, 36 (3), pp.253-266. 10.1051/jphys:01975003603025300 . jpa-00208250

\section{HAL Id: jpa-00208250 https://hal.science/jpa-00208250}

Submitted on 1 Jan 1975

HAL is a multi-disciplinary open access archive for the deposit and dissemination of scientific research documents, whether they are published or not. The documents may come from teaching and research institutions in France or abroad, or from public or private research centers.
L'archive ouverte pluridisciplinaire HAL, est destinée au dépôt et à la diffusion de documents scientifiques de niveau recherche, publiés ou non, émanant des établissements d'enseignement et de recherche français ou étrangers, des laboratoires publics ou privés. 
Classification

Physics Abstracts 8.510

\title{
ORBITAL SUPERLATTICE IN THE DEGENERATE HUBBARD MODEL
}

\author{
M. CYROT and C. LYON-CAEN, \\ Laboratoire de Magnétisme, BP 166 Centre de Tri, 38042 Grenoble-Cedex, France \\ (Reçu le 10 juin 1974, révisé le 25 novembre 1974)
}

\begin{abstract}
Résumé. - En utilisant un modèle de Hubbard deux fois dégénéré nous étudions quelques effets dus à la dégénérescence orbitale dans les bandes étroites. Lorsqu'il y a exactement un électron par atome, l'état de base est ferromagnétique et il se forme un superréseau orbital : le réseau se divise en deux sous-réseaux; sur chacun des sous-réseaux il y a prédominance d'une des deux orbitales. Nous discutons l'importance de l'énergie d'échange intraatomique sur la stabilité de cet état. Nous calculons les excitations collectives de ce système dans l'approximation RPA : nous trouvons en plus des ondes de spin un nouveau type d'excitation lié au superréseau orbital : les ondes orbitales. Nous étudions également l'effet de la température dans l'approximation du champ moléculaire ; le superréseau orbital disparaît toujours à une température supérieure à celle de Curie.
\end{abstract}

\begin{abstract}
We study some effects of the orbital degeneracy in narrow band solids using a doubly degenerate Hubbard model. When there is exactly one electron per atom the ground state is ferromagnetic and an orbital superlattice is set up : the lattice breaks up into two sublattices, each with predominantly one of the orbital states. We discuss the importance of the intraatomic exchange energy for the stability of this state. We calculate the collective excitations corresponding to this ground state in the RPA : we find, besides the spin wave modes, a new kind of excitation linked with the orbital superlattice : the orbital waves. The effect of temperature is investigated in the molecular field approximation : the orbital superlattice disappears at a higher temperature than the Curie temperature.
\end{abstract}

1. Introduction. - Magnetism in transition metals and in their compounds is known to be due to intraatomic Coulomb interaction [1]. The simplest model which takes this into account is that due to Hub-
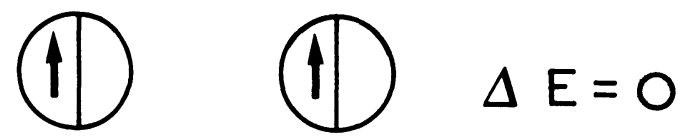
bard [2]. It has been investigated by many authors, but no complete solution exists up to now. This model describes a narrow and non-degenerate tight binding band and permits repulsion between electrons only when they are on the same site. However Van Vleck [3] has emphasized the importance of degeneracy for the occurrence of ferromagnetism. Thus it is of importance to reintroduce it in the simple Hubbard model. In the following our purpose will be to study the effect of degeneracy in this model and to show that completely new features can arise from it.

With one electron per atom, the non degenerate Hubbard hamiltonian probably leads to antiferromagnetism. Van Vleck proposed the following mechanism for ferromagnetism when degeneracy is introduced : consider two weakly interacting atoms each with one electron apiece, the electrons prefer to line up parallel and occupy different orbitals in order to lower their energy by mixing in ionic states with parallel spins; these states lie lowest in energy because of Hund's coupling.
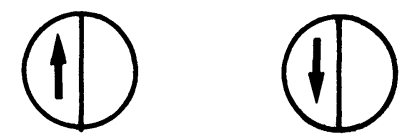

$\Delta E=-\frac{2+2}{u_{1}}$
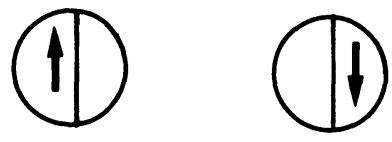

$\Delta E=-\frac{2 r^{2}}{u_{2}}$
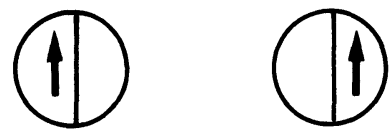

$\Delta E=-\frac{2 r^{2}}{u_{2} J}$

FIG. 1. - Energies of the four possible configurations when $t \ll U$. 
The model we are considering here consists of two degenerate tight binding bands with intraatomic Coulomb interactions. The Coulomb interaction between two electrons depends on the configuration of the two electrons : $U_{1}, U_{2}$ and $J$ are respectively the intraband Coulomb, interband Coulomb and exchange energies. We then suppose that the hopping integrals $t_{i j}$ connect only electrons on neighbouring sites in the same orbital. Following Van Vleck, we consider two atoms with one electron apiece. If $t_{i j}=0$ each electron is localized on an atom. The second order contributions to the energy, due to the virtual transfers, are given in figure 1 for the four electron configurations. The lowest energy state is found to be a ferromagnetic state where the electrons of neighbouring sites are in different orbitals : we have an antiferromagnetic ordering for the orbital states.

Roth [4] suggested that this mechanism works for sufficiently narrow bands : then the most favorable configuration is to form two sublattices each with predominantly one of the orbital states and the spins tend to line up parallel to lower the energy during virtual transfers. However, Roth's formalism describes only the very narrow band limit when all the spins are aligned in the same direction.

In this paper we will develop this natural generalization of ordinary super-exchange which is responsable not only for magnetism but for orbital ordering. We will discuss the ground state, the influence of temperature and the main consequences for physical effect.

We first consider in section 2 the atomic limit. In the non degenerate case, Anderson [5] has shown that the Hubbard band hamiltonian is equivalent to a Heisenberg hamiltonian with an exchange interaction given in terms of the bandwidth and the Coulomb interaction. We work out a similar transformation for the degenerate case. The result is not so simple because the effective hamiltonian describes not only the ordering of spin but also the ordering of orbital states. The lowest energy state is ferromagnetic for spins and antiferromagnetic for orbits. Kügel and Khomskii [6] work out similar limit but our results are somewhat different from these authors because we use a more complete hamiltonian including all spin flip exchange interactions (interband and intraband spin flips).

In section 3 we extend this calculation for finite value of the bandwidth. We use a formalism developed in a previous paper [7] based on a HartreeFock approximation. This formalism is a generalization of the spin dependent potential introduced by Slater. Our potential is not only spin dependent but orbital dependent. Different orbital states see different Hartree-Fock potential. Such a method has been used by Ashkenazi and Weger $[8,9]$ to describe the band structure of $\mathrm{V}_{2} \mathrm{O}_{3}$. Here we point out the close relationship between the magnetic and orbital ordering. This calculation shows that the orbital super- lattice favours the appearence of magnetism. Magnetism can be obtained for values such that the usual Stoner criterium is not fulfilled. We also consider different kinds of orbital order which turn out to be unstable. This result is in agreement with a calculation of Inakagi and Kubo [10] who examined the stability at zero temperature of different types of phases.

The effect of orbital order on the collective excitations is discussed in section 4. We compare the spin wave energies with the energy calculated by Yamada and Shimizu [11] without orbital order. We also point out that there exist other excitations without spin reversing which are linked only with the orbital order.

Section 5 discusses the effect of temperature. The orbital lattice is found to be more stable than ferromagnetism. Thus two transitions occur. At the lower one ferromagnetism disappears and, at the higher, the orbital order. Section 6 gives some experimental consequences of the orbital superlattice and the relationship with Jahn-Teller effect $[12,13]$ is discussed.

2. The atomic limit. -2.1 The HubBard hamilTONIAN. - We consider the Hubbard hamiltonian for a doubly degenerate $E_{\mathbf{g}}$ band [2] in a cubic lattice. It contains two types of terms : the band energy and the intraatomic Coulomb interactions. The general expression for this hamiltonian is :

$$
\begin{gathered}
H=H_{0}+H_{1} \\
H_{1}=\sum_{\substack{i j \\
\sigma m m^{\prime}}} t_{i j}^{m m^{\prime}} C_{i m \sigma}^{+} C_{j m^{\prime} \sigma} \\
H_{0}=\sum_{\substack{i \\
m m^{\prime}}} U_{m m^{\prime}} n_{i m^{\prime} \uparrow} n_{i m^{\prime} \downarrow}+\sum_{i \sigma}\left(U_{12}-J\right) n_{i 1 \sigma} n_{i 2 \sigma}- \\
-J \sum_{i}\left(C_{i 1 \uparrow}^{+} C_{i 1 \downarrow} C_{i 2 \downarrow}^{+} C_{i 2 \uparrow}+\text { C.C. }\right) \\
-J \sum_{i}\left(C_{i 1 \uparrow}^{+} C_{i 2 \uparrow} C_{i 1 \downarrow}^{+} C_{i 2 \downarrow}+\text { C.C. }\right)
\end{gathered}
$$

$m$ and $m^{\prime}$ are the subband indices, and $\sigma$ is the spin indice. $t_{i j}^{m m^{\prime}}$ is the hopping integral between state $m$ on site $i$ and state $m^{\prime}$ on site $j$. We use the tight binding approximation : only the hopping integrals between nearest neighbours are assumed to be non zero. Here for simplicity, we make the following approximations :

- we neglect the hopping integrals $t_{i j}^{12}$ connecting electrons in different orbital states;

- we assume that $t_{i j}^{m m}$ is independent from $m: t_{i j}^{m m}=t_{i j}$;

- we assume that $t_{i j}$ is the same for all directions of the $i-j$ pair.

Then the first part of the hamiltonian has a simple form :

$$
H_{1}=\sum_{\substack{i j \sigma \\ m}} t_{i j} C_{i m \sigma}^{+} C_{j m \sigma}=\sum_{k m \sigma} \varepsilon_{k} C_{k m \sigma}^{+} C_{k m \sigma} .
$$


The band structure depends only on one parameter, the bandwidth:

$$
W=2 z t_{i j} .
$$

This hamiltonian $H_{1}$ is not valid for an $E_{\mathrm{g}}$ band, but we show in the appendix that the qualitative results are not changed when we use the true hopping integrals.

In the second part of the hamiltonian we assume that the intraband Coulomb energies $U_{m m}$ are the same for the two bands : $U_{11}=U_{22}=U_{1}$; we define $U_{12}=U_{2}$. Usually $U_{2} / U_{1}$ is of the order of 1 , $J / U_{2}$ of the order of 0.1 .

In the following we will vary $U_{1} U_{2}$ and $J$ independently for our discussion. However in a realistic model $U_{1} U_{2}$ and $J$ are related as shown in the appendix.

2.2 EFFeCtive hamiltonian. - In the non-degenerate case Anderson [5] has shown that for one electron per atom the effective hamiltonian to second order in $t_{i j}$ is an Heisenberg hamiltonian with an exchange interaction :

$$
J_{i j}=\frac{2 t_{i j}^{2}}{U} .
$$

We can apply the same type of transformation to the degenerate case ; however the effective hamiltonian does not reduce to a simple Heisenberg hamiltonian.

If $t_{i j}=0$ in the case of one electron per atom, each atom has one electron characterized by its spin and orbital state; we call these ground states $\left|\alpha_{i}\right\rangle$. These states form a complete basis for the set of states with one electron per atom. If $t_{i j}=0$, to the lowest order in perturbation the ground state belongs to the $\left|\alpha_{i}\right\rangle$ states subspace. Thus the effective hamiltonian has to verify the equation :

$$
\left\langle\alpha_{i}|H| \alpha_{j}\right\rangle=\left\langle\alpha_{i}\left|H_{\text {eff }}\right| \alpha_{j}\right\rangle+0\left(t_{i j}^{3}\right)
$$

for all $\left|\alpha_{i}\right\rangle$ and $\left|\alpha_{j}\right\rangle$ states.

To calculate $H_{\text {eff }}$ we use the following method [16] : if we can find a transformation $S$ which is a solution for the equation :

$$
H_{1}+\left[H_{0}, S\right]=0
$$

the hamiltonian $\widetilde{H}=\mathrm{e}^{-S} H \mathrm{e}^{S}$ is equivalent to $H$ and does not contain any term of the first order in $H_{1}$ :

$$
\tilde{H}=H_{0}+\frac{1}{2}\left[H_{1}, S\right]+0\left(H_{1}^{3}\right) .
$$

If we restrict ourselves to the states with one electron per atom we can find the transformation $S$ from eq. (5). Eq. (4) becomes :

$$
\begin{aligned}
\left\langle\alpha_{i}\left|H_{\text {eff }}\right| \alpha_{j}\right\rangle & =\left\langle\alpha_{i}\left|S H_{0} S\right| \alpha_{j}\right\rangle \\
& =\sum_{\beta}\left\langle\alpha_{i}\left|S H_{0}\right| \beta\right\rangle\left\langle\beta|S| \alpha_{j}\right\rangle
\end{aligned}
$$

where $|\beta\rangle$ is any eigenstate of $H_{0}$.
Using (5) it is easy to show that :

$$
\left\langle\alpha_{i}\left|S H_{0}\right| \beta\right\rangle=\left\langle\alpha_{i}\left|H_{1}\right| \beta\right\rangle .
$$

Then we can restrict the states $|\beta\rangle$ to a particular subspace : $\left\langle\alpha_{i}\left|H_{1}\right| \beta\right\rangle$ is non zero only if $|\beta\rangle$ is a state where one electron has hopped from one atom to a neighbouring one : these states have one atom without any electron and one atom with two electrons. They are not eigenstates of $\boldsymbol{H}_{0}$. However, eq. (7) remains true. Defining $E_{\beta}, \lambda_{\beta}$, and $\left|\beta^{\prime}\right\rangle$ for each $|\beta\rangle$ state as in table I, we can write :

$$
\langle\beta| H_{0}=E_{\beta}\langle\beta|-\lambda_{\beta}\left\langle\beta^{\prime}\right| .
$$

TABLE I

Values of $E_{\beta}$ and $\lambda_{\beta}$
Configuration

of the 2 elec-

trons atom in the $|\beta\rangle$ state

$\begin{array}{ccc}\overline{\text { (11) }} & - & - \\ \text { (11) } & U_{2} & J \\ \text { (11) } & U_{2}-J & 0 \\ U_{1} & J\end{array}$

Configuration of the 2 electrons atom in the $\left|\beta^{\prime}\right\rangle$ state<smiles></smiles><smiles>[18OH]</smiles>

Using (9) and (5), $\left\langle\beta|S| \alpha_{j}\right\rangle$ is of the form :

$$
\left\langle\beta|S| \alpha_{j}\right\rangle=-\left\langle\beta\left|X H_{1}\right| \alpha_{j}\right\rangle
$$

where $X$ is an operator.

Substituting (9) and (10) into (7), we obtain :

$$
\left\langle\alpha_{i}\left|H_{\text {eff }}\right| \alpha_{j}\right\rangle=-\left\langle\alpha_{i}\left|H_{1} X H_{1}\right| \alpha_{j}\right\rangle
$$

where in $H_{1} X H_{1}$ we keep only terms

$$
t_{i j}^{2} C_{i m \sigma}^{+} C_{j m \sigma} X_{j} C_{j m^{\prime} \sigma^{\prime}}^{+} C_{i m^{\prime} \sigma^{\prime}}
$$

which contribute to $H_{\text {eff }}$. These terms take into account the virtual hopping between neighbouring sites and the intraatomic spin flip in the intermediate state which has one doubly occupied site.

We obtain the following expression for $H_{\text {eff }}$ :

$$
\begin{aligned}
H_{\mathrm{eff}}= & -\sum_{\substack{i j \\
m \\
\sigma \neq \sigma^{\prime}}} \frac{U_{1} t_{i j}^{2}}{U_{1}^{2}-J^{2}}\left(n_{i m \sigma} n_{j m \sigma^{\prime}}\right. \\
& \left.-C_{i m \sigma}^{+} C_{i m \sigma^{\prime}} C_{j m \sigma^{\prime}}^{+} C_{j m \sigma}\right) \\
& -\sum_{\substack{i j \\
m \neq m^{\prime} \\
\sigma \neq \sigma^{\prime}}} \frac{J t_{i j}^{2}}{U_{1}^{2}-J^{2}}\left(C_{i m \sigma}^{+} C_{i m^{\prime} \sigma} C_{j m \sigma^{\prime}}^{+} C_{j m^{\prime} \sigma^{\prime}}\right. \\
& \left.-C_{i m \sigma}^{+} C_{i m^{\prime} \sigma^{\prime}} C_{j m \sigma^{\prime}}^{+} C_{j m^{\prime} \sigma}\right) \\
& -\sum_{\substack{i j \\
m \neq m^{\prime} \\
\sigma \neq \sigma^{\prime}}} \frac{U_{2} t_{i j}^{2}}{U_{2}^{2}-J^{2}}\left(n_{i m \sigma} n_{j m^{\prime} \sigma^{\prime}}\right. \\
& \left.-C_{i m \sigma}^{+} C_{i m^{\prime} \sigma^{\prime}} C_{j m^{\prime} \sigma^{\prime}}^{+} C_{j m \sigma}\right)
\end{aligned}
$$




$$
\begin{aligned}
& +\sum_{\substack{i j \\
m \neq m^{\prime} \\
\sigma \neq \sigma^{\prime}}} \frac{J t_{i j}^{2}}{U_{2}^{2}-J^{2}}\left(C_{i m \sigma}^{+} C_{i m^{\prime} \sigma} C_{j m^{\prime} \sigma^{\prime}}^{+} C_{j m \sigma^{\prime}}\right. \\
& \left.-C_{i m \sigma}^{+} C_{i m \sigma^{\prime}} C_{j m^{\prime} \sigma^{\prime}}^{+} C_{j m^{\prime} \sigma}\right) \\
& -\sum_{\substack{i j \\
m \neq m^{\prime} \\
\sigma}} \frac{t_{i j}^{2}}{U_{2}-J}\left(n_{i m \sigma} n_{j m^{\prime} \sigma}\right. \\
& \left.-C_{i m \sigma}^{+} C_{i m^{\prime} \sigma} C_{j m^{\prime} \sigma}^{+} C_{j m \sigma}\right) .
\end{aligned}
$$

Kugel and Khomskii [6] did not obtain exactly the same expression : in their calculation they considered only intraband spin flip and neglected the last term of $H_{0}$ :

$$
-\sum_{i} J\left(C_{i 1 \uparrow}^{+} C_{i 2 \uparrow} C_{i 1 \downarrow}^{+} C_{i 2 \downarrow}+\text { C.C. }\right)
$$

Thus their effective hamiltonian can be used to calculate the intraband spin waves but not the interband spin waves (Section 4).

2.3 MAgNetic AND ORbital ORDER. - We now want to describe an approximate ground state for the effective hamiltonian. For this we take as the energy of a state $\left|\alpha_{i}\right\rangle$ the following expression :

$$
E_{i}=\left\langle\alpha_{i}\left|H_{\text {eff }}\right| \alpha_{i}\right\rangle=\left\langle\alpha_{i}\left|H_{\mathrm{d}}\right| \alpha_{i}\right\rangle
$$

where $H_{\mathrm{d}}$ is the diagonal part of $H_{\text {eff }}$ (the states $\left|\alpha_{i}\right\rangle$ are not rigorously eigenstates of $H_{\mathrm{eff}}$, but the problem is the same for the antiferromagnetic ground state of an Heisenberg hamiltonian : we neglect the zero point energy of the collective excitations).

By introducing two Ising variables on each site :

- a spin $S_{i}= \pm 1$

- an orbital momentum $L_{i}= \pm 1, L_{i}=+1$ for one of the orbital states, $L_{i}=-1$ for the other, we can write $H_{\mathrm{d}}$ as follows :

$$
\begin{aligned}
H_{\mathrm{d}}= & -\sum_{i j} \frac{t_{i j}^{2}}{4\left(U_{2}^{\prime}-J^{\prime}\right)}\left(1-L_{i} L_{j}\right)\left(1+S_{i} S_{j}\right) \\
& -\sum_{i j} \frac{t_{i j}^{2}}{4 U_{2}^{\prime}}\left(1-L_{i} L_{j}\right)\left(1-S_{i} S_{j}\right) \\
& -\sum_{i j} \frac{t_{i j}^{2}}{4 U_{1}^{\prime}}\left(1+L_{i} L_{j}\right)\left(1-S_{i} S_{j}\right)
\end{aligned}
$$

with

$$
\begin{aligned}
& U_{1}^{\prime}=U_{1}\left[1-\left(\frac{J}{U_{1}}\right)^{2}\right] \\
& U_{2}^{\prime}=U_{2}\left[1-\left(\frac{J}{U_{2}}\right)^{2}\right] \\
& J^{\prime}=J\left[1-\frac{J}{U_{2}}\right] .
\end{aligned}
$$

We can use this expression to calculate the energies of the four configurations shown in figure $1: U_{1} U_{2}$ and $J$ are now replaced by $U_{1}^{\prime} U_{2}^{\prime}$ and $J^{\prime}$; the perturbation theory does not take the spin flip interactions into account. One of the effects of these interactions is to replace the Coulomb and exchange energies by the effective values $U_{1}^{\prime} U_{2}^{\prime}$ and $J^{\prime}$.

However this effect is only of the second order in $J / U$; by neglecting spin flip interactions we can calculate the energy of the ground state up to the first order in $J / U$.

The ground state of $H_{\mathrm{d}}$ is given by the conditions :

$$
\left.\begin{array}{l}
S_{i} S_{j}=1 \\
L_{i} L_{j}=-1
\end{array}\right\} \text { for all } i-j \text { pairs }
$$

The $L_{i} L_{j}=-1$ condition can be fulfilled for all pairs in a simple cubic or bcc lattice, but not in a fcc lattice. In a fcc lattice the ground state is more complicated and we restrict ourselves to simple cubic lattices. In this case the lattice breaks up into two sublattices and one of the orbital states is occupied on each of them.

If $J=0$ the spin order can be either ferromagnetic or antiferromagnetic. Both states are degenerate. The exchange energy stabilizes the ferromagnetic state.

The orbital order is a direct consequence of the degeneracy. Even if $J=0$ the orbital order is antiferromagnetic; this is easy to see from expression (13) when $U_{1}>U_{2}$. In the case when $U_{1}=U_{2}$ and $J=0$ we have three degenerate ordered states at $T=0 \mathrm{~K}$ :

- ferromagnetic with antiferromagnetic orbital order $\left(L_{i} L_{j}=-1, S_{i} S_{j}=+1\right)$;

- antiferromagnetic with antiferromagnetic orbital $\operatorname{order}\left(L_{i} L_{j}=-1, S_{i} S_{j}=-1\right)$;

- antiferromagnetic with ferromagnetic orbital $\operatorname{order}\left(L_{i} L_{j}=1, S_{i} S_{j}=-1\right)$.

But as soon as temperature increases the only stable state is the second one. Thus in the ground state, the orbital order is always antiferromagnetic.

3. The case of a finite bandwidth. - In the previous section we have discussed the atomic limit and the ground state in this limit. We want to generalize our result to the case of finite bandwidth. For this purpose we use a Hartree-Fock approximation which permits the self-consistent potential to be spin and orbital dependent. Such a procedure has been used by Ashkenzi and Weger for $\mathrm{V}_{2} \mathrm{O}_{3}$. We will also assume and verify later that the magnetic order remains ferromagnetic and the orbital order antiferromagnetic. Our Hartree-Fock approximation may be justified for small value of the bandwidth. However it is obvious that near the appearence of magnetism or of orbital order, fluctuations will turn out to be important. Thus we cannot hope for better than a qualitative understanding in this region. 
3.1 METHOD AND RESULTS. - We shall use a selfconsistent approximation, thus the spin flip terms can be neglected. We rewrite the other terms of the intraatomic Coulomb interaction (2) in the following form :

$$
\begin{aligned}
H_{0}=\frac{2 U_{2}-J}{4} \sum_{i} & \left(\sum_{m} n_{i m \sigma}\right)^{2}- \\
& -\frac{J}{4} \sum_{i}\left(\sum_{m} n_{i m \uparrow}-n_{i m \downarrow}\right)^{2} \\
& -\frac{2 U_{2}-U_{1}-J}{4} \sum_{i m}\left(n_{i m \uparrow}+n_{i m \downarrow}\right)^{2} \\
& -\frac{U_{1}-J}{4} \sum_{i m}\left(n_{i m \uparrow}-n_{i m \downarrow}\right)^{2} .
\end{aligned}
$$

We make a Hartree-Fock approximation which introduces four parameters on each site. These parameters will be selfconsistently determined :

$$
\mu_{i m}=\left\langle n_{i m \uparrow}-n_{i m \downarrow}\right\rangle
$$

the magnetic moment due to electrons in orbital $m$,

$$
v_{i m}=\left\langle n_{i m \uparrow}+n_{i m \downarrow}\right\rangle
$$

the number of electrons in orbital $m$.

As we always restrict ourselves to the case of one electron per atom, $v_{i m}$ satisfies the relation $\sum_{m} v_{i m}=1$. The total moment on site $i$ is :

$$
\mu_{i}=\sum_{m} \mu_{i m} .
$$

As we are looking for an orbital superlattice, $\mu_{i m}$ and $v_{i m}$ can be different on each site. In the case of an antiferromagnetic orbital order, the $v_{i m}$ have the following behaviour :

$$
\begin{aligned}
& v_{i 1}=\frac{1}{2}+v_{0} \mathrm{e}^{i \mathbf{p R _ { i }}} \\
& v_{i 2}=\frac{1}{2}-v_{0} \mathrm{e}^{\mathbf{i} \mathbf{R}_{i}}
\end{aligned}
$$

where $\mathbf{p}$ is half a reciprocal vector which divides the lattice sites into two sublattices. Values of $v_{0}$ different from zero describe an orbital superlattice. In the atomic limit we have $v_{0}=\frac{1}{2}$, i.e. $v_{i m}$ is 1 or 0 . For a finite bandwidth, $v_{0}<\frac{1}{2}$ : the two orbitals are occupied on each site but the number of electrons in the two orbitals is different. If there is no orbital order $v_{0}=0$.

The contributions of each orbital to the magnetic moment, $\mu_{i m}$, have the same type of variation. Here we are looking for a ferromagnetic solution; the total magnetic moment $\mu_{i}$ is the same on each site. Then we can write for the magnetic moments :

$$
\begin{aligned}
\mu_{i 1} & =\mu_{0}+\mu_{1} \mathrm{e}^{i \mathbf{p R _ { i }}} \\
\mu_{i 2} & =\mu_{0}-\mu_{1} \mathrm{e}^{i \mathbf{p R _ { i }}} \\
\mu_{i} & =2 \mu_{0} .
\end{aligned}
$$

Using the expressions (16) and (17) in the HartreeFock approximation of (15), the total hamiltonian $H_{0}+H_{1}$ can be exactly diagonalized [17].

For simplicity we assume $U_{1}=U_{2}=U$. The eigenvalues for $\sigma$ spin electrons are independent from $m$ :

$$
\begin{aligned}
& E_{\sigma}^{ \pm}(k)=\frac{\varepsilon_{k}+\varepsilon_{k+p}}{2}-\sigma \mu_{0} \frac{U+J}{2} \pm \frac{1}{2} \times \\
& \times \sqrt{\left(\varepsilon_{k}-\varepsilon_{k+p}\right)^{2}+(U-J)^{2}\left(v_{0}+\sigma \mu_{1}\right)^{2}} .
\end{aligned}
$$

Each of these bands is defined in half of the Brillouin zone and contains one electron per atom if $v_{0}+\sigma \mu_{1} \neq 0$. When $v_{0}+\sigma \mu_{1}=0$ eq. (18) becomes :

$$
E_{\sigma}(k)=\varepsilon_{k}-\sigma \mu_{0} \frac{U+J}{2} .
$$

$E_{\sigma}$ is defined in all the Brillouin zone and contains two electrons per atom.

The total energy is :

$$
\begin{array}{r}
E=N\left[\frac{U+J}{2} \mu_{0}^{2}+\frac{U-J}{2}\left(\mu_{1}^{2}+v_{0}^{2}+\frac{1}{4}\right)+\frac{U}{4}\right]+ \\
+\sum_{k, \lambda} E_{\lambda}(k) n\left(E_{\lambda}\right) .
\end{array}
$$

$n(E)$ is the Fermi function at $T=0 \mathrm{~K} . v_{0}, \mu_{0}$ and $\mu_{1}$ are calculated by minimizing the total energy. Thus we obtain :

$$
\begin{gathered}
\mu_{1}=v_{0}, \\
\frac{1}{U-J}=\frac{1}{N} \sum_{k}^{\prime} \frac{n\left(E_{\uparrow}^{-}\right)-n\left(E_{\uparrow}^{+}\right)}{\sqrt{\left(\varepsilon_{k}-\varepsilon_{k+p}\right)^{2}+4 \mu_{1}^{2}(U-J)^{2}}}, \\
2 \mu_{0}=\frac{1}{N} \sum_{k}^{\prime} n\left(E_{\uparrow}^{-}\right)+n\left(E_{\uparrow}^{+}\right)-\frac{1}{N} \sum_{k} n\left(E_{\downarrow}\right)
\end{gathered}
$$

$\left(\sum_{k}^{\prime}=\right.$ sum over half of the Brillouin zone; we chose the normalization so that $\left.\sum_{k}^{\prime}=N\right)$.

The relation (21) between $v_{0}$ and $\mu_{1}$ is very simple : it is a consequence of the assumption $U_{1}=U_{2}$. If $v_{0}=\mu_{1}$ the energies for down spin electrons form only one band which contains two electrons per atom. - The band of down spin electrons is degenerate, but this degeneracy would be lifted if $U_{1} \neq U_{2}$. If $U_{1}=U_{2}$ a down spin electron interacts with an up spin electron in the same way when the two electrons are in the same or in different orbitals.

We can solve (22) and (23) in the atomic limit $\varepsilon_{k}=0$. In this limit we find that the solution of the lowest energy is $\mu_{0}=\frac{1}{2}, \mu_{1}=\frac{1}{2}$; in accordance with the results of section 2 ; we have three energy levels :

$$
E_{\uparrow}^{-}=0 \quad E_{\uparrow}^{+}=U-J \quad E_{\downarrow}=U .
$$

If $W$ is very small, the three bands are very near these three levels (Fig. 3); in this situation the band $E_{\uparrow}$ 


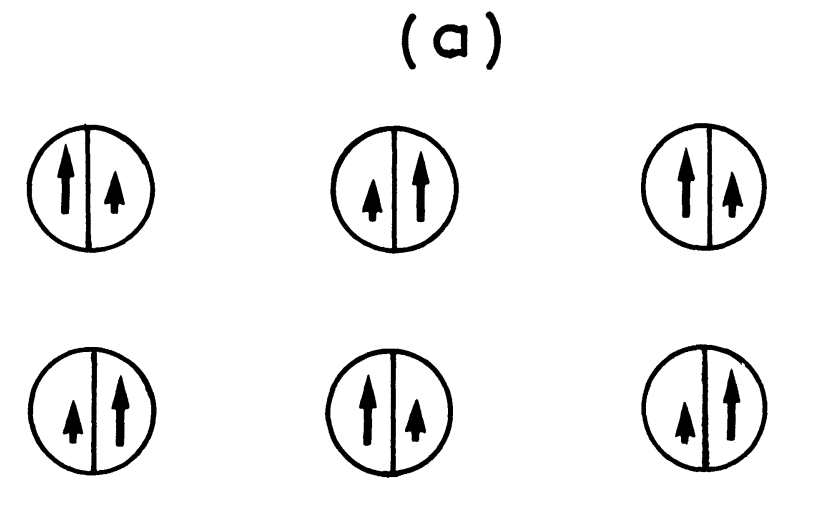

(b)
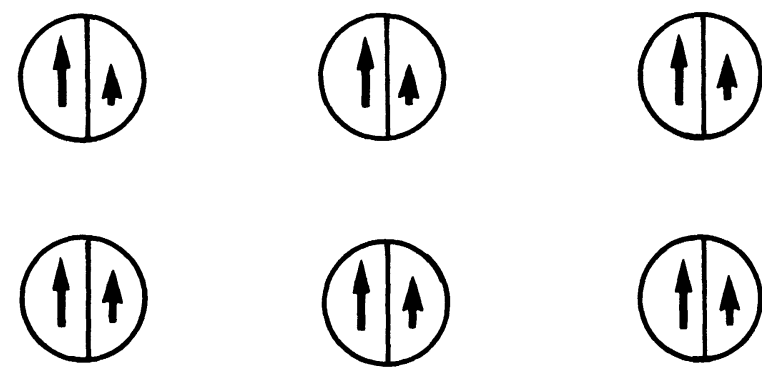

FIG. 2. - Two types of orbital orders : (a) Antiferromagnetic orbital order ; (b) Ferromagnetic orbital order.

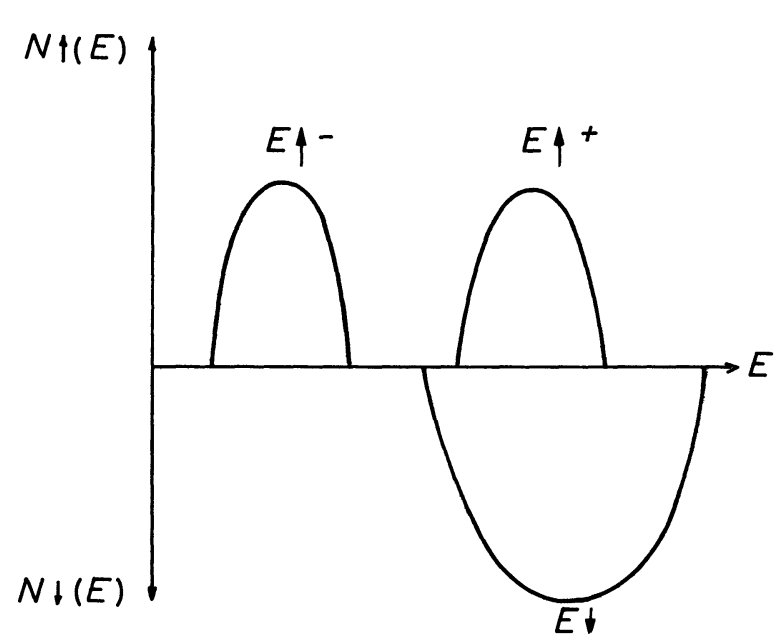

FIG. 3. - Schematic density of states for spin up and spin down electrons.

is completely filled and the other bands empty. Thus we have a ferromagnetic insulator with a magnetic moment on each site $2 \mu_{0}=1$; the occupation difference between the two subbands is always smaller than 1 as can be seen from (22). For a very narrow band, we can develop (22) :

$$
\mu_{1}=\frac{1}{2}\left[1-\frac{2 z t^{2}}{(U-J)^{2}}\right] .
$$

The metal non metal transition occurs when the $E_{\uparrow}^{+}$band or the $E_{\downarrow}$ band overlap the $E_{\uparrow}^{-}$band. If the $E_{\uparrow}^{+}$band overlaps first, the total moment $2 \mu_{0}$ does not decrease at the transition. If the $E_{\downarrow}$ overlaps first the moment decreases at the transition; this is always the case in the simple cubic structure.

Figure 4 gives the values of $\mu_{0}$ and $\mu_{1}$ as a function of $U / W$ for different values of $J / U$ in the simple cubic structure.

- For large values of $J / U(J / U>0,5)$ magnetism appears when $U / W$ is larger than a critical value, and the orbital superlattice appears at a larger critical value. Both transitions are second order.
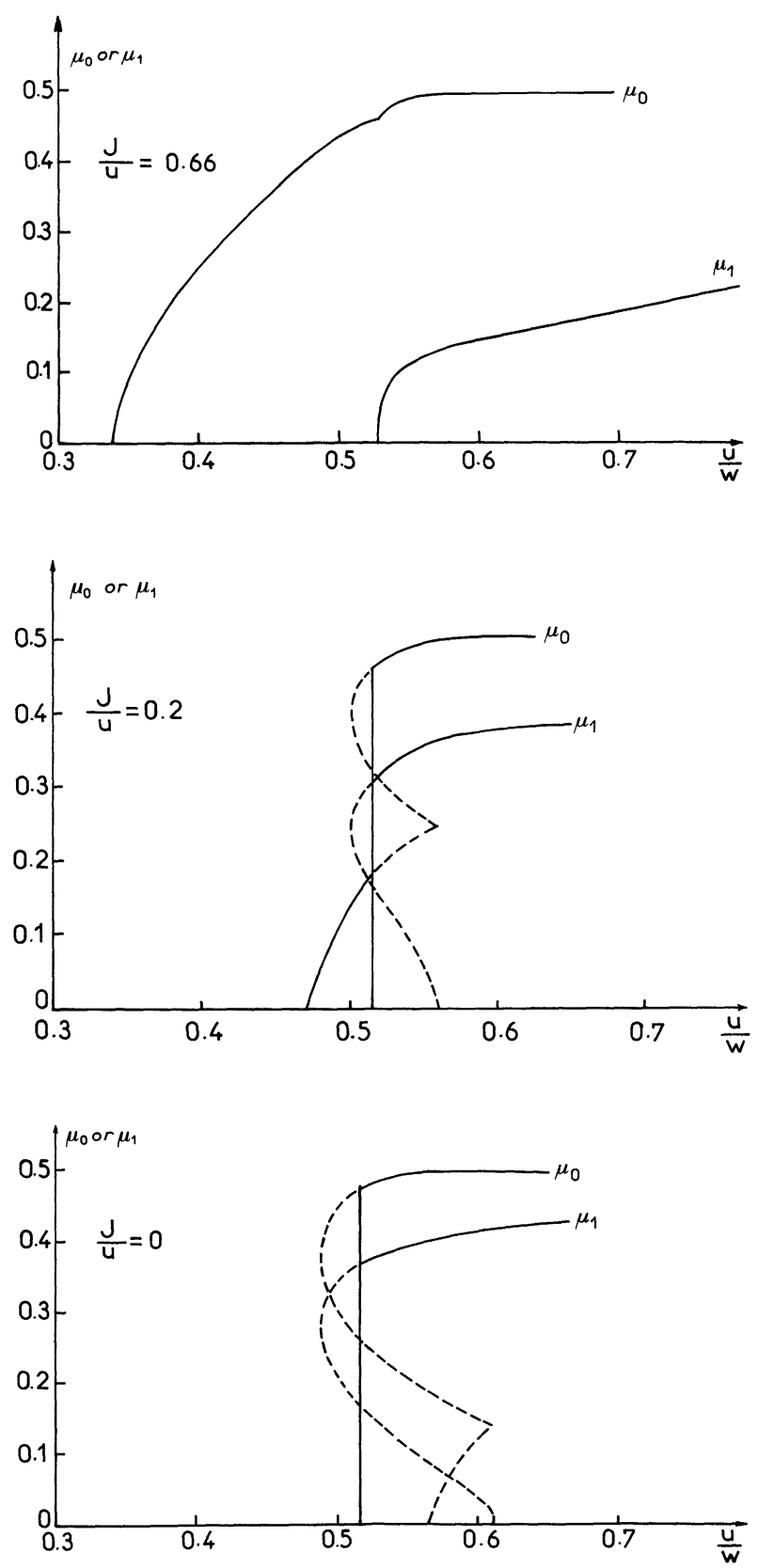

FIG. 4. - Magnetic moment on a site $2 \mu_{0}$, and difference of occupation of the two subbands on a site $2 \mu_{1}$ as a function of $U / W$ for : (a) large; (b) intermediate; (c) low values of $J / U$. 
- For small values of $J / U(J / U<0,2)$ magnetism and orbital superlattice appear for the same value of $U / W$ with a discontinuous transition. The transition occurs when two different solutions have the same energy. The energy of these solutions is given by (20). The difference in energy between the non magnetic state $\left(\mu_{0}=\mu_{1}=0\right)$ and a state where $\mu_{0}$ and $\mu_{1}$ are non zero for a fixed value of $U / W$ is given by :

$$
\begin{aligned}
\Delta E & =-\frac{W}{4} \int_{0}^{(U / W)_{0}} \times \\
\times & {\left[4\left(1-\frac{J}{U}\right) \mu_{1}^{2}+\left(2-\frac{J}{U}\right) \mu_{0}^{2}\right] \mathrm{d}\left(\frac{U}{W}\right) . }
\end{aligned}
$$

This expression can be obtained from (20) by calculating the derivative $\partial E / \partial U$ for constant $W$ and $J$.

- For intermediate values of $J / U$ magnetism appears continuously and there is a discontinuity when the orbital superlattice appears.

3.2 Stability of THE solution. - The condition for appearance of a magnetic moment $\mu_{0}$ can be obtained from (23) :

$$
(U+J) \rho\left(E_{\mathrm{F}}\right) \geqslant 1
$$

where $\rho\left(E_{\mathrm{F}}\right)$ is the density of state at Fermi energy. This criterion is a generalization of the Stoner criterion for the degenerate case [18]. We can obtain the condition for appearance of an orbital superlattice form eq. (22) :

$$
(U-J) \sum_{k}^{\prime} \frac{n\left(E_{\uparrow}^{-}\right)-n\left(E_{\uparrow}^{+}\right)}{\left|\varepsilon_{k}-\varepsilon_{k+p}\right|} \geqslant 1 .
$$

$\sum_{k}^{\prime} \frac{n\left(E_{\uparrow}^{-}\right)-n\left(E_{\uparrow}^{+}\right)}{\left|\varepsilon_{k}-\varepsilon_{k+p}\right|}$ is the susceptibility of the up spin band for wave vector $q=p$, and $\rho\left(E_{\mathrm{F}}\right)$ is the susceptibility of the two bands for wave vector $q=0$. We can rewrite (27) as :

$$
(U-J) \chi_{\uparrow}\left(E_{\mathbf{F}}\right) \geqslant 1 .
$$

In (26) $\rho\left(E_{\mathrm{F}}\right)$ is the density of states for $\mu_{0}=0$ but it depends on $\mu_{1}$. In (28) $\chi_{\uparrow}\left(E_{\mathrm{F}}\right)$ is calculated for $\mu_{1}=0$ but it is function of the number of electrons in the spin up band, or a function of $\mu_{0}$.

The criterions (26) and (28) are similar to those obtained by Coqblin and Blandin [18] for the degenerate Anderson model : their condition for appearance of spin magnetism is exactly (26). For orbital magnetism their condition is :

$$
(U-J) \rho_{\uparrow}\left(E_{\mathrm{F}}\right) \geqslant 1
$$

where $\rho_{\uparrow}$ is the state density of up spin electrons; it is also the susceptibility of the spin up band for $q=0$ and (29) is the condition for appearance of a ferro- magnetic orbital order. Coqblin and Blandin [18] also found first or second order transitions according to the value of $J / U$ : one first order transition for small values of $J / U$ and two second order transitions for large values of $J / U$ as we have found here.

The first order transition occurs at a value of $U / W$ where the condition (26) is not fulfilled for $\mu_{1}=0$ as can be seen on figure 4 . Thus the orbital superlattice stabilizes the ferromagnetic state. However, the orbital superlattice is stable only if $U-J$ is large enough; if $U=J$ spin up electrons do not interact if they are in different orbitals. Thus it is not favourable to create a dissymmetry in the occupation of the two orbitals on one atom. When $J / U$ is large the superlattice is stable only for large values of $U / W$; on the other hand eq. (26) shows that the higher $J / U$ is, the smaller the value of $U / W$ for which the ferromagnetic state becomes stable. Thus the two transitions are distinct. For small values of $J / U$ conditions (26) and (28) may be fulfilled with $\mu_{0}$ and $\mu_{1}$ both different from zero while they are not fulfilled for $\mu_{0}=\mu_{1}=0$ because $\rho\left(E_{\mathrm{F}}\right)$ and $\chi_{\uparrow}\left(E_{\mathrm{F}}\right)$ are increasing functions of $\mu_{0}$ and $\mu_{1}$.

The exchange energy is not favourable to the orbital order. In section 2 we already noticed that the orbital order is a consequence of degeneracy but not of exchange energy. However, exchange energy is necessary to have a ferromagnetic state : we can show that the energy difference between the ferromagnetic and the antiferromagnetic states (both with antiferromagnetic orbital order) is zero when $J=0$ and increases with $J$. For small values of $J$ :

$$
E_{\mathrm{AF}}-E_{\mathrm{F}} \simeq J\left(\mu_{0}^{2}-\mu_{1}^{2}\right)
$$

The preceding results show that $\mu_{0}$ is always larger than $\mu_{1}$. Thus the ferromagnetic state is more stable when $J$ is large.

4. Collective excitations. - In a non degenerate band the only collective excitations are acoustical spin waves. In the case of two degenerate bands we will obtain several kinds of excitations.

In the atomic limit we can describe a solid by a set of atoms with an electron apiece, this electron being in a state $m \sigma$. An electron in the state $1 \uparrow$ in the ground state can be excited in three different states :

- in state $1 \downarrow$ : the corresponding collective excitations are intraband spin waves;

- in state $2 \downarrow$ : it gives interband spin waves

— in state $2 \uparrow$ : it leads to a new kind of collective excitations, the orbital waves.

In the atomic limit there is always one electron per atom for all these excitations; thus we can use the hamiltonian $H_{\text {eff }}$ obtained in section 2 .

We find the same kinds of excitations when using the band picture. If $U / W$ is large enough only two 
of the six bands are filled : $E_{\uparrow 1}^{-}$and $E_{\uparrow 2}^{-}$. The other $E_{\uparrow 1}^{+} \quad E_{\uparrow 2}^{+} \quad E_{\downarrow 1}$ and $E_{\downarrow 2}$ are empty (in section 3 we ommitted the subband indices because the energies do not depend on these indices; but here we need the expressions of the wave functions which depend on these indices).

An electron in the $E_{\uparrow 1}^{-}$band can be excited in four different bands :

- in the $E_{\uparrow 1}^{+}$band : it leads to plasmons,

- in the $E_{\uparrow 2}^{+}$band : it leads to orbital waves,

- in the $E_{\downarrow 1}$ band : it leads to intraband spin waves,

— in the $E_{\downarrow 2}$ band : it leads to interband spin waves.

In this section we will study the spin waves and the orbital waves using both atomic and band descriptions. All calculations are made in the simple cubic structure.

4.1 INTRABAND SPIN WAVES. - These spin waves arise from reversing the spin of an electron while keeping it in the same orbital. This excitation is represented by an operator $S^{-}$defined by :

$$
S^{-}=\sum_{i m} a_{i m} S_{i m}^{-}
$$

where $S_{i m}^{-}=C_{i m \downarrow}^{+} C_{i m \uparrow}$. The spin waves energies are determined using the random phase approximation (RPA). In the atomic limit, we can use the effective hamiltonian (12). We find two normal modes :

- The acoustical mode is given by $S_{q_{1}}^{-}+S_{q_{2}}^{-}$; the spin waves in the two subbands are in phase. For small $q$ the excitation energy is :

$$
E=D q^{2} \quad \text { with } \quad D=\frac{2 J t^{2} a^{2}}{U_{2}^{2}-J^{2}}
$$

- The optical mode is given by $S_{q_{1}}^{-}-S_{q_{2}}^{-}$; the spin waves formed in the two subbands are out of phase. The energy of this mode, for $q=0$, is :

$$
E=\frac{24 J t^{2}}{U_{2}^{2}-J^{2}}
$$

In the case of a finite bandwidth we must use the full hamiltonian. We need the expression of the operators corresponding to the eigenstates. Using the same notation as des Cloizeaux [17, 19] these operators are :

- for $1 \uparrow$ states :

$E_{1 \uparrow}^{-}$band : $\quad \alpha_{k 1 \uparrow}^{+}=\cos \varphi_{k} C_{k 1 \uparrow}^{+}+\sin \varphi_{k} C_{k+p 1 \uparrow}^{+}$

$E_{1 \uparrow}^{+}$band : $\beta_{k 1 \uparrow}^{+}=\mathrm{e}^{i \lambda k}\left(\cos \varphi_{k} C_{k+p 1 \uparrow}^{+}-\sin \varphi_{k} C_{k 1 \uparrow}^{+}\right)$.

$\lambda$ is such as $\mathrm{e}^{i \lambda P}=-1$.

$\cos ^{2} \varphi_{k}=\frac{1}{2}\left[1+\frac{\varepsilon_{k+p}-\varepsilon_{k}}{\sqrt{\left(\varepsilon_{k+p}-\varepsilon_{k}\right)^{2}+4(U-J)^{2} \mu_{1}^{2}}}\right]$
- for $2 \uparrow$ states :

$E_{2 \uparrow}^{-}$band : $\quad \alpha_{k 2 \uparrow}^{+}=\mathrm{e}^{i \lambda k}\left(\sin \varphi_{k} C_{k+p 2 \uparrow}^{+}-\cos \varphi_{k} C_{k 2 \uparrow}^{+}\right)$ $E_{2 \uparrow}^{+}$band : $\beta_{k 2 \uparrow}^{+}=\sin \varphi_{k} C_{k 2 \uparrow}^{+}+\cos \varphi_{k} C_{k+p 2 \uparrow}^{+}$;

- for $\downarrow$ spin states the operators are simply the Bloch operators $C_{k m \downarrow}^{+}$.

We restrict ourselves to the $\mu_{0}=\frac{1}{2}$ case ; only $\overline{E_{m} \uparrow}$ bands are occupied. Thus the intraband spin waves are defined by the operator :

$$
S_{q}^{-}=\sum_{k} \lambda_{k 1} C_{k+q 1 \downarrow}^{+} \alpha_{k 1 \uparrow}+\lambda_{k 2} C_{k+q 2 \downarrow}^{+} \alpha_{k 2 \uparrow} .
$$

In the RPA we get $2 N$ linear equations in $\lambda_{k 1}$ and $\lambda_{k 2}$. The solutions can be separated into two groups :

$N$ solutions are given by $\lambda_{k 1}=\lambda_{k 2}=\lambda_{k}$ and :

$$
\begin{gathered}
\lambda_{k}[E(k+q) \downarrow-E(k) \uparrow-E]=\frac{U+J}{N} \cos \varphi_{k} \sum_{k^{\prime}} \times \\
\times \lambda_{k^{\prime}} \cos \varphi_{k^{\prime}}+\frac{U-J}{N} \sin \varphi_{k} \sum_{k^{\prime}} \lambda_{k^{\prime}} \sin \varphi_{k^{\prime}}
\end{gathered}
$$

The $N$ other solutions are given by $\lambda_{k 1}=-\lambda_{k 2}=\mu_{k}$ and :

$$
\begin{gathered}
\mu_{k}[E(k+q) \downarrow-E(k) \uparrow-E]=\frac{U-J}{N} \cos \varphi_{k} \sum_{k^{\prime}} \times \\
\times \mu_{k^{\prime}} \cos \varphi_{k^{\prime}}+\frac{U+J}{N} \sin \varphi_{k} \sum_{k^{\prime}} \mu_{k^{\prime}} \sin \varphi_{k^{\prime}}
\end{gathered}
$$

We can notice that if $J=0$ (35) and (36) are identical : in this case there is no coupling between the two subbands.

Eq. (35) gives the optical mode. Before calculating the spin wave energy we solve (35) when $J=0$. In this case one of the $N$ solutions, for $q=0$, is given by $\lambda_{k}=\cos \varphi_{k}$ and the energy of this solution is $E=0$. The other solutions are single particule excitations.

If $J \neq 0$ (35) has no solution with zero energy; thus the collective excitation given by

$$
\lambda_{k 1}=\lambda_{k 2}=\cos \varphi_{k}
$$

is an optical mode. For small $J$ we can calculate the energy of this mode :

$$
E=J\left(1-4 \mu_{1}^{2}\right) .
$$

When $U / W \gg 1, \mu_{1}$ is given by (24) and (37) is identical to (32) at first order in $J / U$.

Expression (37) is valid for all values of $\mu_{1}$. In particular if there is no orbital order i.e. $\mu_{1}=0$ we find $E=J$. This is the value found by Yamada and Shimizu [11], Chao [20], Chang and Young [21] for the optical mode in a degenerate band without orbital order. 
We consider now the solution of eq. (36). When $q=0$, one of the solutions is given by $\lambda_{k}=\cos \varphi_{k}$, $E=0$ for all values of $J$; thus this mode is the acoustical mode. For small $q$ we can obtain the dispersion relation from (36) :

$E=\frac{2}{N} \sum_{k}\left[\cos ^{2} \varphi_{k}\left(\varepsilon_{k+q}-\varepsilon_{k}\right)-\frac{\left(\varepsilon_{k+q}-\varepsilon_{k}\right)^{2}}{2 U}\right]$.

The first term is exact but the second one is calculated up to the lowest order in $t / U$.

If $t / U \ll 1$, (38) becomes :

$$
E=\frac{2 t^{2} a^{2} J}{U(U-J)} q^{2} .
$$

Again this is identical, up to the first order in $J / U$, to the result (31) found in the atomic limit.

Roth [4] calculated the next term of the development in $t / U$, which is negative; thus if $J=0$, the spin wave energy is negative and the ferromagnetic state is unstable. The ferromagnetic state is stable if $J$ is higher than a critical value.

4.2 INTERBAND SPIN WAVES. - In these excitations the magnetic order and the orbital order are destroyed simultaneously. The spin wave operator is defined as :

$$
S^{-}=\sum_{i m} a_{i m} S_{i m}^{-}
$$

with $S_{i m}^{-}=C_{i m^{\prime} \downarrow}^{+} C_{i m \uparrow}\left(m \neq m^{\prime}\right)$.

In the atomic limit, we use $H_{\text {eff }}$ to calculate the spin wave modes. In the RPA we get for the energies of the normal modes :

$$
E=2 z t^{2}\left(\frac{1}{U_{2}-J}-\frac{1}{U_{1}-J}\right)
$$

and

$$
E=2 z t^{2}\left(\frac{1}{U_{2}-J}-\frac{1}{U_{1}+J}\right) .
$$

If $U_{1}=U_{2}$ we find only two spin wave energies, an acoustical and an optical one. Each of them is twicely degenerate.

If $U_{1} \neq U_{2}$ the four spin waves, an acoustical and three optical ones, have different energies (Fig. 5). When $J / U \ll 1$ and $\frac{U_{1}-U_{2}}{U} \ll 1$, the optical modes have the following energies :

$$
\left.\begin{array}{ll}
E_{1} \simeq \frac{4 z J t^{2}}{U^{2}} & \text { (intraband spin waves) } \\
E_{2} \simeq 2 z \frac{\left(U_{1}-U_{2}\right) t^{2}}{U^{2}} \\
E_{3} \simeq 2 z\left(U_{1}-U_{2}+2 J\right) \frac{t^{2}}{U^{2}}
\end{array}\right\} \text { (interband spin waves). }
$$

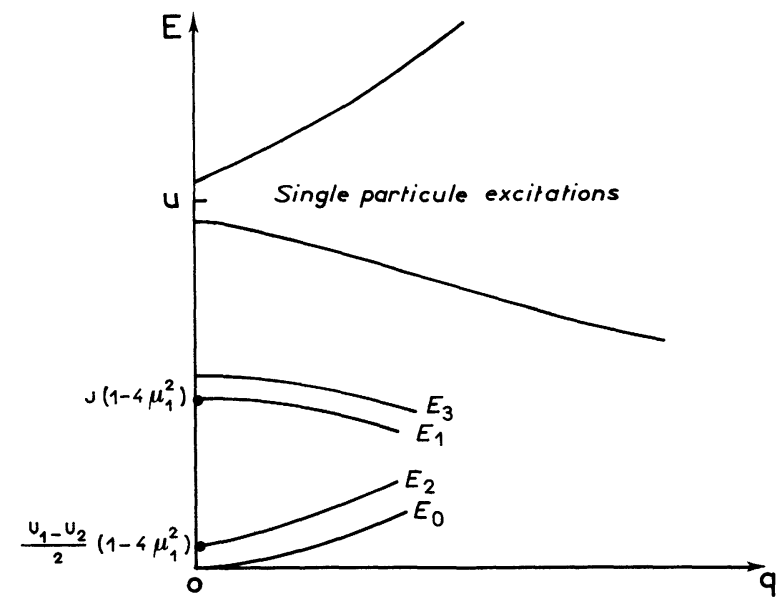

FIG. 5. - Schematic curves of the spin wave spectrum : $E_{0}$ and $E_{1}$ are the intraband spin waves, $E_{2}$ and $E_{3}$ the interband spin waves.

For a finite bandwidth, the spin wave operator is defined by :

$$
S_{q}^{-}=\sum_{k} \lambda_{k 1} C_{k+q 2 \downarrow}^{+} \alpha_{k 1 \uparrow}+\lambda_{k 2} C_{k+q 1 \downarrow}^{+} \alpha_{k 2 \uparrow} .
$$

If $U_{1}=U_{2}$, the $2 N$ equations in $\lambda_{k 1}$ and $\lambda_{k 2}$ are exactly the same as for the intraband spin waves. Thus if $U_{1}=U_{2}$ the two branches are degenerate.

When $U_{1} \neq U_{2}$, by extrapolating the expressions (41) we can write :

$$
\begin{aligned}
& E_{2}=\frac{U_{1}-U_{2}}{2}\left(1-4 \mu_{1}^{2}\right) \\
& E_{3}=\left(\frac{U_{1}-U_{2}}{2}+J\right)\left(1-4 \mu_{1}^{2}\right) .
\end{aligned}
$$

These expressions are valid if $U_{1}=U_{2}$. If $U_{1} \neq U_{2}$ they give the correct result (41) when $U / W \gg 1$. On the other hand they give also the correct result when there is no orbital order : for this case the spin wave energies have been calculated by Yamada and Shimizu [11] who found for the optical modes :

$E_{1}=2 \mu_{0} J$ (intrabạnd spin waves)

$\left.\begin{array}{l}E_{2}=\left(U_{1}-U_{2}\right) \mu_{0} \\ E_{3}=\left(U_{1}-U_{2}+2 J\right) \mu_{0}\end{array}\right\}$ (interband spin waves).

When $\mu_{0}=\frac{1}{2}$ (44) gives the same results as (43) and (37) with $\mu_{1}=0$.

4.3 Orbital waves. - In this type of excitation the magnetic order is not destroyed, but the orbital order is destroyed. We always restrict ourselves to the case $\mu_{0}=\frac{1}{2}$ in which there are only up spin electrons.

In the atomic limit we can restrict ourselves to the following terms of $H_{\text {eff }}$ :

$$
\begin{aligned}
H_{\mathrm{orb}}=-\sum_{i j} & \frac{t_{i j}^{2}}{U_{2}-J}\left[n_{i 1 \uparrow} n_{j 2 \uparrow}+n_{i 2 \uparrow} n_{j 1 \uparrow}-\right. \\
& \left.-\left(C_{i \uparrow \uparrow}^{+} C_{i 2 \uparrow} C_{j 2 \uparrow}^{+} C_{j 1 \uparrow}+\text { C.C. }\right)\right] .
\end{aligned}
$$


This can be written :

$$
H_{\text {orb }}=-\sum_{i j} \frac{t_{i j}^{2}}{U_{2}-J}\left(\frac{1}{2}-2 \mathbf{L}_{i} \mathbf{L}_{j}\right) .
$$

$\mathbf{L}_{i}$ is an orbital momentum :

$$
\begin{aligned}
& L_{i z}=\frac{1}{2}\left(n_{i 1 \uparrow}-n_{i 2 \uparrow}\right) \\
& L_{i}^{+}=C_{i 1 \uparrow}^{+} C_{i 2 \uparrow} \\
& L_{i}^{-}=C_{i 2 \uparrow}^{+} C_{i 1 \uparrow} .
\end{aligned}
$$

$H_{\text {orb }}$ is a Heisenberg hamiltonian with an antiferromagnetic interaction; in the ground state

$$
L_{i z}=\frac{1}{2} \mathrm{e}^{i p R_{i}}
$$

as we found previously. We can also use $H_{\text {orb }}$ to calculate the orbital waves. The dispersion relation is similar to antiferromagnetic spin waves :

$$
E=C q \quad \text { with } \quad C=\frac{4 \sqrt{3} t^{2} a}{U_{2}-J} .
$$

In the general case of a finite bandwidth, from the Hubbard hamiltonian we can also deduce an $H_{\text {orb }}$ by keeping the terms only for up spin electrons :

$$
H_{\text {orb }}=\sum_{k m} \varepsilon_{k} n_{k m}+\left(U_{2}-J\right) \sum_{i m} n_{i 1} n_{i 2} .
$$

We can omit the spin indices because only one spin direction is involved. (48) is equivalent to the non degenerate Hubbard hamiltonian and we get the orbital waves when using the same calculations as for the spin waves in the antiferromagnetic Hubbard model. Thus we can use the result of des Cloizeaux [19] : the excitation energies are solutions of the equation :

$$
F q(E) G q(E)=[H q(E)]^{2}
$$

where the functions $F_{q}, G_{q}$ and $H_{q}$ have exactly the same expression as in [19].

At $q=0$ one of the solutions is $E=0$ : this is an acoustical mode. The other solutions are single particules excitations.

For small $q$ (49) gives $E=C q$ and if $\frac{U-J}{W} \gg 1$ we find for $C$ the previous expression (47).

At low temperature the variations of $\mu_{0}$ and $\mu_{1}$ are determined by the collective excitations. The magnetic moment $2 \mu_{0}$ decreases as $T^{3 / 2}$; this is a consequence of the dispersion relation of the acoustical spin wave $E=D q^{2}$. The dispersion relation of the orbital wave is linear in $q$; thus the orbital order decreases like $T^{2}$ at low temperature.

5. Effect of temperature. - In this section, we discuss the effect of temperature. We restrict ourselves to the atomic limit where we can use the effective hamiltonian $H_{\text {eff }}$ derived in section 2 . This limit also allows us to neglect single electron dynamic effects which exist when there is a free Fermi surface. This effect can be important as soon as the subbands overlap. The molecular field approximation that we will use in this section allows us to obtain qualitative results concerning magnetic and orbital orders.

We use expression (13) to calculate the partition function in the molecular field approximation :

$$
Z=\operatorname{tr} \mathrm{e}^{-\beta H_{\mathrm{d}}} \text {. }
$$

We rewrite $H_{\mathrm{d}}$ as :

$H_{\mathrm{d}}=\sum_{i j} J_{1} S_{i} S_{j}+\sum_{i j} J_{2} L_{i} L_{j}+\sum_{i j} J_{3} L_{i} S_{i} L_{j} S_{j}$

with :

$$
\begin{aligned}
& J_{1}=\frac{t_{i j}^{2}}{4}\left(\frac{1}{U_{1}^{\prime}}+\frac{1}{U_{2}^{\prime}}-\frac{1}{U_{2}^{\prime}-J^{\prime}}\right) \\
& J_{2}=\frac{t_{i j}^{2}}{4}\left(-\frac{1}{U_{1}^{\prime}}+\frac{1}{U_{2}^{\prime}}+\frac{1}{U_{2}^{\prime}-J^{\prime}}\right) \\
& J_{3}=\frac{t_{i j}^{2}}{4}\left(\frac{1}{U_{1}^{\prime}}-\frac{1}{U_{2}^{\prime}}+\frac{1}{U_{2}^{\prime}-J^{\prime}}\right)
\end{aligned}
$$

$J_{2}$ and $J_{3}$ are always positive. $J_{1}$ may be negative if $\frac{J^{\prime}}{U_{2}^{\prime}}>\frac{U_{2}^{\prime}}{U_{1}^{\prime}+U_{2}^{\prime}}$. In any case, $J_{1}<J_{3}<J_{2}$.

In the molecular field approximation $H_{\mathrm{d}}$ has the following form :

$$
\begin{aligned}
H_{\mathrm{d}}=\sum_{i j} 2 J_{1}\left\langle S_{j}\right. & S_{i}+2 J_{2}\left\langle L_{j}\right\rangle L_{i}+ \\
& +2 J_{3}\left\langle L_{j} S_{j}\right\rangle L_{i} S_{i} \\
& -\sum_{i j} J_{1}\left\langle S_{j}\right\rangle\left\langle S_{i}\right\rangle \\
& +J_{2}\left\langle L_{j}\right\rangle\left\langle L_{i}\right\rangle \\
& +J_{3}\left\langle L_{i} S_{i}\right\rangle\left\langle L_{j} S_{j}\right\rangle .
\end{aligned}
$$

We have introduced three molecular fields : $J_{1}\left\langle S_{j}\right\rangle, J_{2}\left\langle L_{j}\right\rangle$ and $J_{3}\left\langle L_{j} S_{j}\right\rangle .\left\langle S_{j}\right\rangle$ is the total moment on site $j$, $\left\langle L_{j}\right\rangle$ is the difference between the occupations of the two orbitals, $\left\langle L_{j} S_{j}\right\rangle$ is the difference between the magnetic moments of the two orbitals. These three parameters are determined selfconsistently by minimizing the free energy $F=-k T \log Z$.

At $T=0 \mathrm{~K}$ the ground state is ferromagnetic with antiferromagnetic orbital order. Thus we have to look for a solution which has the following behaviour :

$$
\begin{aligned}
\left\langle S_{j}\right\rangle & =S \\
\left\langle L_{j}\right\rangle & =L \mathrm{e}^{i \mathbf{p R} j} \\
\left\langle L_{j} S_{j}\right\rangle & =P \mathrm{e}^{i \mathbf{p R} j} .
\end{aligned}
$$

We obtain three equations to calculate $S, L$ and $P$. These equations have many solutions for the same value of the parameters $J_{1}, J_{2}, J_{3}$. We can show that 
there are at most two transition temperatures because two parameters always vanish together, and the third one generally becomes zero at a higher temperature.

As in our case $J_{1}<J_{3}<J_{2}$, when the temperature decreases, the first transition occurs at $k T_{0}=2 z J_{2}$; at this temperature, the parameter $L$ becomes different from zero : $T_{0}$ is the orbital temperature for which an orbital superlattice is set up. The parameters $S$ and $P$ become different from zero below the Curie temperature $T_{\mathrm{C}}$ : We obtain :

$k T_{\mathrm{C}}=\frac{4 z J_{1} J_{3}\left(1-L^{2}\right)}{\sqrt{\left(J_{3}-J_{1}\right)^{2}+4 J_{1} J_{3}\left(1-L^{2}\right)}-\left(J_{3}-J_{1}\right)}$

where

$$
L=\operatorname{th} \frac{2 z J_{2} L}{k T_{\mathrm{C}}}
$$

The results are shown on figure 6.

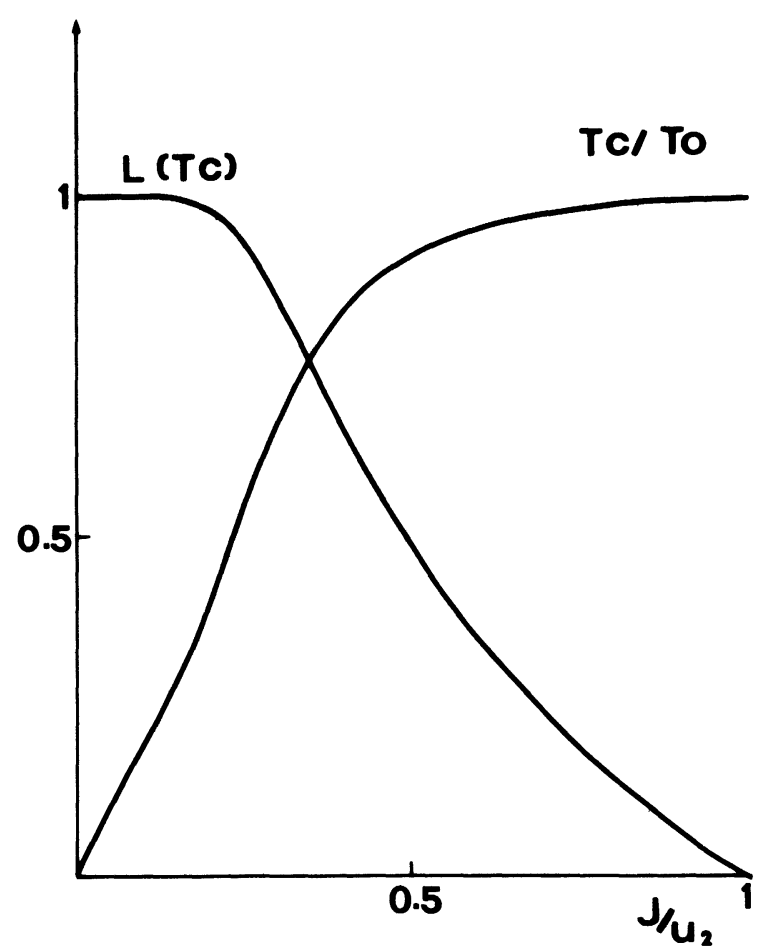

Fig. 6. - Curie temperature $T_{\mathrm{C}}$ and value of the parameter $L$ at the magnetic transition for $U_{2} / U_{1}=0,9$.

The Curie temperature is zero when $J=0$. For small values of $J / U$, the transition temperature is given by $k T_{\mathrm{C}}=2 z\left(J_{3}-J_{1}\right)$ and the transition occurs when the orbital order is not destroyed $(L \simeq 1$ at the transition). Thus in this case, the Curie temperature is given by the exchange interaction between the spins calculated for $L=1$ because $L$ does not change in the whole magnetic region; this interaction is exactly :

$$
J_{3}-J_{1} \simeq \frac{z J t^{2}}{U_{2}^{2}}
$$

for small values of $J / U$.
For larger values of $J / U$, the transition occurs when $L$ is much smaller than 1 (Fig. 6) : the exchange energy between the spins depends on the temperature, and it decreases when temperature increases ; between two atoms, when $L_{i} L_{j}=-1$ the exchange is ferromagnetic and given by $J_{3}-J_{1}$, but when $L_{i} L_{j}=+1$ the exchange between two atoms is antiferromagnetic and given by $J_{3}+J_{1}$; thus when the temperature increases some of the interactions become antiferromagnetic and the effective ferromagnetic exchange energy decreases.

The orbital temperature $T_{0}$ is always determined by the parameter $J_{2}$ which is the exchange energy between the $L_{i}$ when the magnetic order is destroyed $(S=P=0)$. For small values of $J / U$ and for $U_{2} / U_{1} \simeq 1$ we obtain :

$$
k T_{0}=\frac{z t^{2}}{2 U} .
$$

Thus in this case :

$$
\frac{T_{\mathrm{C}}}{T_{0}} \simeq \frac{2 J}{U}
$$

For small values of $J / U$ the two transitions are second order; we can show that near $T_{\mathrm{C}}$ :

$$
S \simeq P \simeq \text { th } 2 z \beta\left(J_{3}-J_{1}\right) S .
$$

The behaviour of $L, S$ and $P$ is shown on figure 7 .

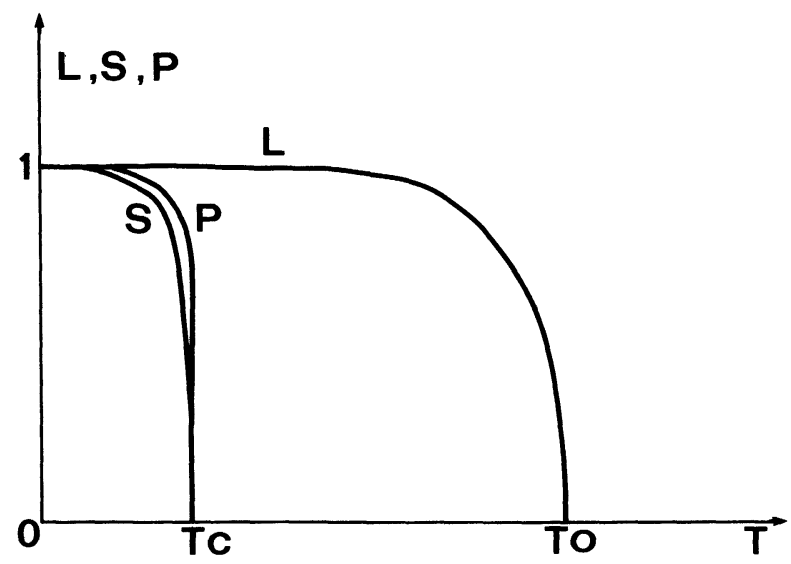

Fig. 7. - Schematic behaviour of the three parameters $L, S$ and $P$ as a function of temperature for small values of $J / U$.

For large values of $J / U$ the transition can be first order. We have determined the order of the transition for the different values of $U_{2} / U_{1}$ and of $J / U_{2}$ by an expansion of the free energy near the Curie temperature.

The results are shown on figure 8 . When the transition is first order the Curie temperature is not given by eq. (52) which is valid only for second order transitions. 


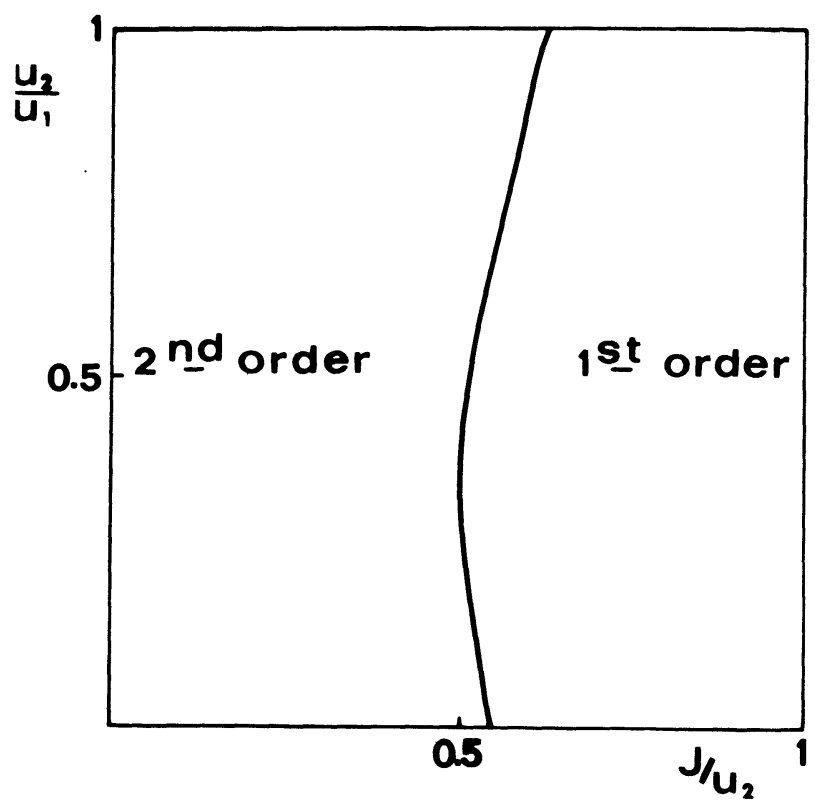

FIG. 8. - Order of the magnetic transition for the different values of $U_{2} / U_{1}$ and $J / U_{2}$.

In conclusion, the molecular field approximation gives us only qualitative results. For instance we have shown that the orbital transition always occurs at a higher temperature than the magnetic transition. For small values of $J / U$ the Curie temperature is much lower than the orbital temperature $\left(T_{\mathrm{C}} / T_{0}=2 \mathrm{~J} / U\right)$. This result does not depend on the approximation we used.

6. Direct effects of the orbital superlattice. - We have shown, using a very simple model, that an important effect due to degeneracy is the formation of an orbital superlattice. This superlattice may be detected by $\mathbf{X}$ rays or neutron diffraction experiments : on the two sublattices the electrons have different wave functions and the form factors are different. This gives additionnal diffraction peaks as for an antiferromagnet. These peaks exist in $\mathrm{KCuF}_{3}$ [22] even above the magnetic transition temperature; they indicate an orbital ordering. However $\mathrm{KCuF}_{3}$ is an insulator and in this case the orbital ordering can arise from a Jahn Teller effect as well as the mechanism we discussed here [6].

In the Jahn-Teller effect, the orbital degeneracy is lifted by a deformation of the lattice. The lattice distorts near a transitional ion and the elastic interactions between local distortion are responsible for the cooperative effect. Orbital superlattice is a consequence of distortions and magnetic ordering is not in general related to the orbital superlattice. In our approach, orbital ordering and magnetism are related and are due to a superexchange mechanism. This mechanism is independent of any distortion. However orbital ordering can be coupled to the lattice and produce a distortion. If such a distortion occurs, our mechanism is difficult to distinguish from a Jahn Teller one. Thus it would be interesting to find such an orbital ordering in metallic compounds where the Jahn Teller effect is not expected to play a significant role. Cobalt disulfide seems to be a good candidate : the study of the transition metal disulfides, $\mathrm{FeS}_{2}, \mathrm{CoS}_{2}, \mathrm{NiS}_{2}, \mathrm{CuS}_{2}, \mathrm{ZnS}_{2}$ [23], shows that this series corresponds to the filling of an $E_{\mathrm{g}}$ band; in the first elements of the series $\left(\mathrm{FeS}_{2}, \mathrm{CoS}_{2}\right.$ and $\left.\mathrm{NiS}_{2}\right)$ there is no mixing of the $E_{\mathrm{g}}$ band with $\mathrm{s}$ ou p bands, thus our approach could be relevant for these compounds.

Another consequence of the orbital superlattice is the very low value of the spin wave energies; the optical mode energies are reduced by a factor $\left(1-4 \mu_{1}^{2}\right)$ when an orbital ordering is present; the acoustical mode also has a low energy : without orbital order the $D$ coefficient is proportional to $t$; we found here that $D$ is proportional to $t^{2} J / U_{2}^{2}$. The acoustical spin wave energy is much reduced by this effect.

The orbital waves contribute to the specific heat as well as the spin waves; the contribution of the orbital waves gives a $T^{3}$ term while the spin wave contribution gives a $T^{3 / 2}$ term. Both contributions can be separated.

When we try to apply our crude theory to real systems we have to take into account some important features neglected here. The first one is the structure. Here we have only considered simple cubic structure; in $\mathrm{CoS}_{2}$ the cobalt atoms are on a fcc lattice; in this structure the orbital superlattice will be more complicated because it is not possible for all pairs of nearest neighbours to be antiferromagnetic. Another important parameter to consider is the hopping integral $t_{i j}^{12}$ which connects electrons in different orbitals. These terms have an important effect upon the stability of the superlattice. However we show in the appendix that the orbital order has still an important role in the stability of the ferromagnetic state.

\section{APPENDIX}

Orbital and magnetic order in an $\boldsymbol{E}_{\mathrm{g}}$ band. - In the preceding sections we have used a very simple model for the two degenerate states. In the case of two $E_{\mathrm{g}}$ states (which is the case in $\mathrm{KCuF}_{3}$ and in $\mathrm{CoS}_{2}$ ) some of our assumptions have to be modified. The model is more complicated but in the atomic limit we can obtain some results in the same way as in section 2 .
The two $E_{\mathrm{g}}$ wave functions are :

$$
\begin{aligned}
& \varphi_{1}=\left(x^{2}-y^{2}\right) f(r) \\
& \varphi_{2}=\frac{1}{\sqrt{3}}\left(2 z^{2}-x^{2}-y^{2}\right) f(r) .
\end{aligned}
$$

Using these expressions of the wave functions the parameters $U_{m m^{\prime}}$ and $J_{m m^{\prime}}$ have the following 
expressions :

$$
\begin{array}{rl}
U_{m m^{\prime}}=\int\left|\varphi_{m}\left(r_{1}\right)\right|^{2} & V\left(r_{1}-r_{2}\right)\left|\varphi_{m^{\prime}}\left(r_{2}\right)\right|^{2} \times \\
\times \mathrm{d} v_{1} \mathrm{~d} v_{2} . \\
J=\int \varphi_{1}\left(r_{1}\right) \varphi_{2}\left(r_{2}\right) V\left(r_{1}-r_{2}\right) \times \\
\times \varphi_{1}\left(r_{2}\right) \varphi_{2}\left(r_{1}\right) \mathrm{d} v_{1} \mathrm{~d} v_{2} .
\end{array}
$$

Generally there are other intraatomic terms in the degenerate Hubbard hamiltonian (1) as :

$$
I_{m m^{\prime}} C_{i m \uparrow}^{+} C_{i m \downarrow}^{+} C_{i m^{\prime} \uparrow} C_{i m \downarrow},
$$

with :

$I_{m m^{\prime}}=\int\left|\varphi_{m}\left(r_{1}\right)\right|^{2} V\left(r_{1}-r_{2}\right) \varphi_{m}\left(r_{2}\right) \varphi_{m^{\prime}}\left(r_{2}\right) \mathrm{d} v_{1} \mathrm{~d} v_{2}$

but for the $E_{\mathrm{g}}$ wave function $I_{m m^{\prime}}=0$.

The $t_{i j}^{m m^{\prime}}$ elements of the hopping matrix $T_{i j}$ depend

\begin{tabular}{|c|c|c|c|}
\hline $\begin{array}{c}\text { Direction of } \\
\text { the } i-j \text { pair }\end{array}$ & $O x$ & Oy & $O z$ \\
\hline- & - & - & - \\
\hline$t_{i j}^{11}$ & $\frac{3}{2} t$ & $\frac{3}{2} t$ & 0 \\
\hline$t_{i j}^{22}$ & $\frac{1}{2} t$ & $\frac{1}{2} t$ & $2 t$ \\
\hline$t_{i j}^{12}$ & $\frac{\sqrt{3}}{2} t$ & $-\frac{\sqrt{3}}{2} t$ & 0 \\
\hline
\end{tabular}
on the direction of the $i-j$ pair. They are different in the three directions [24] (Table II).

\section{TABLE II}

Hopping integrals for the $E_{\mathrm{g}}$ states [24]

We can write the Hubbard hamiltonian in another basis defined by the two wave functions :

$$
\begin{aligned}
& \psi_{1}=\cos \theta \varphi_{1}+\sin \theta \varphi_{2} \\
& \psi_{2}=-\sin \theta \varphi_{1}+\cos \theta \varphi_{2}
\end{aligned}
$$

In this new basis the Coulomb energies are $U_{m m^{\prime}}(\theta)$, $J(\theta), I_{m m^{\prime}}(\theta)$. These energies can be calculated using the expressions (A.1), (A.2) and (A.3) with $\psi_{m}$ instead of $\varphi_{m}$. Using the general expression for the Coulomb energies it is easy to see that

$$
\frac{U_{11}(\theta)+U_{22}(\theta)}{2}+J(\theta) \text { and } U_{12}(\theta)-J(\theta)
$$

are independent of $\theta$.

In the case of the $E_{\mathrm{g}}$ wave functions, all Coulomb interactions are independent of $\theta$.
For $\theta=\pi / 3$ we find :

$$
\begin{aligned}
& \psi_{1}=\left(z^{2}-x^{2}\right) f(r) \\
& \psi_{2}=\frac{1}{\sqrt{3}}\left(2 y^{2}-x^{2}-z^{2}\right) f(r) .
\end{aligned}
$$

Thus, in this basis, $\psi_{1}$ and $\psi_{2}$ have the same form as $\varphi_{1}$ and $\varphi_{2}$, and the Coulomb interactions are the same in the two basis; using the relations between $U_{m m^{\prime}}(\pi / 3), J(\pi / 3), I_{m m^{\prime}}(\pi / 3)$ and $U_{m m^{\prime}}(0), J(0), I_{m m^{\prime}}(0)$ we find :

$$
U_{11}=U_{22}=U_{1}
$$

and if $U_{12}=U_{2}$

$$
U_{1}-U_{2}=2 J
$$

If we put (A.4) and (A.5) in the general expressions we see that $U_{m m^{\prime}}(\theta), J(\theta)$ and $I_{m m^{\prime}}(\theta)$ are independent of $\theta$.

Thus for the $E_{\mathrm{g}}$ wave functions, the Coulomb interactions do not depend on the choice of the wave functions and we have very simple relations (A.4) and (A.5) between the Coulomb energies.

Relation (A.5) shows that it is not possible to make the assumption $U_{1}=U_{2}$ as we did in this paper. However the effect of the exchange energy $J$ is much more important than that of the difference $U_{1}-U_{2}$.

The intraatomic terms of the hamiltonian (1) are invariant with respect to a wave function rotation, but the hopping terms are not invariant. However the trace and the determinant of the hopping matrix $T_{i j}$ are independent of $\theta$ and for the $E_{\mathrm{g}}$ wave functions independent of the direction of the $i-j$ pair.

$$
\begin{aligned}
& T=\operatorname{tr} T_{i j}=t_{i j}^{11}+t_{i j}^{22} \\
& D=\operatorname{det} T_{i j}=t_{i j}^{11} t_{i j}^{22}-\left(t_{i j}^{12}\right)^{2} .
\end{aligned}
$$

In order to find the ground state of an $i-j$ pair of atoms in the atomic limit we have to calculate the energies of the four configurations (Fig. 1) in an arbitrary basis :

1) The ferromagnetic state with antiferromagnetic orbital order $\left(S_{i} S_{j}=+1, L_{i} L_{j}=-1\right)$ has an energy :

$$
E_{1}(\theta)=-\frac{\left(t^{11}\right)^{2}+\left(t^{22}\right)^{2}}{U_{2}-J}
$$

The minimum of $E_{1}(\theta)$ occurs in the basis where $\left|t^{11}(\theta)\right|^{2}+\left|t^{22}(\theta)\right|^{2}$ is maximum.

As $\left|t^{11}(\theta)\right|^{2}+\left|t^{22}(\theta)\right|^{2}=T^{2}-2 D-2\left|t^{12}(\theta)\right|^{2}$, the minimum of $E_{1}$ is :

$$
E_{1}^{\min }=-\frac{T^{2}-2 D}{U_{2}-J} .
$$

The best basis to form the orbital superlattice is the basis in which the matrix $T$ is diagonal, but in 
this basis $t^{11}(\theta) \neq t^{22}(\theta)$. In this paper we assumed that $t^{11}=t^{22}$ and $t^{12}=0$ : in this case all orbital superlattices are stable because $T$ is always diagonal.

For two atoms, the orbital superlattice has to be formed in the basis where $t^{12}=0$. But in a crystal it is not possible that $t_{i j}^{12}(\theta)=0$ for all directions of the $i$-j pair.

2) The energy of the antiferromagnetic state with an antiferromagnetic orbital order

$$
\left(S_{i} S_{j}=-1, L_{i} L_{j}=-1\right)
$$

is :

$$
\begin{aligned}
& E_{2}(\theta)=-\frac{\left(t^{11}\right)^{2}+\left(t^{22}\right)^{2}}{U_{2}}-2 \frac{\left(t^{12}\right)^{2}}{U_{1}} \\
& E_{2}(\theta)=-\frac{T^{2}-2 D}{U_{2}}-2\left(t^{12}\right)^{2}\left(\frac{1}{U_{1}}-\frac{1}{U_{2}}\right) .
\end{aligned}
$$

$E_{2}$ is minimum when $t^{\mathbf{1 2}}(\theta)=0$. The two states with $L_{i} L_{j}=-1$ are stable in the same basis, and in this basis $E_{2}^{\min }>E_{1}^{\min }$. Thus the ferromagnetic state is more stable than the antiferromagnetic one.

3) The energy of the antiferromagnetic state with ferromagnetic orbital order $\left(S_{i} S_{j}=-1, L_{i} L_{j}=+1\right)$ is :

$$
\begin{aligned}
& E_{3}(\theta)=-\frac{\left(t^{11}\right)^{2}+\left(t^{22}\right)^{2}}{U_{1}}-\frac{2\left(t^{12}\right)^{2}}{U_{2}} \\
& E_{3}(\theta)=-\frac{T^{2}-2 D}{U_{1}}-2\left(t^{12}\right)^{2}\left(\frac{1}{U_{2}}-\frac{1}{U_{1}}\right)
\end{aligned}
$$

$E_{3}(\theta)$ is the average energy for the two equivalent configurations.

The minimum of $E_{3}$ occurs when $t^{12}$ is maximum, i.e. when

$$
\operatorname{tg} 2 \theta=-\frac{t^{11}-t^{22}}{2 t^{12}}
$$

and in this basis $\left|t^{12}(\theta)\right|^{2}=\frac{1}{4}\left(T^{2}-4 D\right)$.

In all bases $E_{3}(\theta)>E_{2}(\theta)$ and the difference $E_{3}-E_{2}$ is always proportional to $U_{1}-U_{2}$.
Thus in all bases the antiferromagnetic state is more stable if there is an orbital order.

4) The last configuration is ferromagnetic with ferromagnetic orbital order

$$
\left(S_{i} S_{j}=+1, L_{i} L_{j}=+1\right) .
$$

Its energy is :

$$
E_{4}(\theta)=-\frac{2\left(t^{12}\right)^{2}}{U_{2}-J}
$$

The minimum occurs when $t^{12}$ is maximum and :

$$
E_{4}^{\min }=-\frac{T^{2}-4 D}{2\left(U_{2}-J\right)} .
$$

This state is never stable because $E_{4}^{\min }>E_{1}^{\min }$. Moreover in all basis $E_{4}(\theta)>E_{1}(\theta)$ because $E_{1}^{\max }<E_{4}^{\min }$.

For two atoms, the ground state is always the first one. In a crystal it is more complicated because the hopping integrals are different in the three directions. Kugel and Khomskii [6] used the following method in the case of a simple cubic lattice : on both sublattices the basis can be different; these are defined by $\theta$ and $\theta^{\prime}$. On one sublattice the occupied state is $\psi_{1}=\cos \theta \varphi_{1}+\sin \theta \varphi_{2}$ and on the other one it is $\psi_{2}=\cos \theta^{\prime} \varphi_{1}+\sin \theta^{\prime} \varphi_{2}$. The authors calculate the crystal energy for several spin configurations and minimize this energy according to $\theta$ and $\theta^{\prime}$. Thus they find for small values of $J / U$ :

$$
\theta=\frac{\pi}{3} \text { and } \theta^{\prime}=-\frac{\pi}{3} .
$$

In the $(x-y)$ plane the orbital order is antiferromagnetic and the spin order is ferromagnetic. Along the $z$ axis they found two degenerate configurations, both with antiferromagnetic spin order but where the orbital order can be either ferro- or antiferromagnetic. This is only a consequence of their assumption $U_{1}=U_{2}$ because in this case $E_{2}(\theta)=E_{3}(\theta)$ in all basis. But if $U_{1} \neq U_{2}$ only one of these states is stable : the state with antiferromagnetic orbital order.

\section{References}

[1] Slater, J. C., Phys. Rev. 49 (1936) 537.

[2] Hubbard, J., Proc. R. Soc. A 276 (1963) 238; ibid. A 277 (1964) 237.

[3] Van Vleck, J. H., Rev. Mod. Phys. 25 (1953) 220.

[4] Roth, L. M., Phys. Rev. 149 (1966) 306; J. Appl. Phys. 38 (1967) 1065.

[5] Anderson, P. W., Sol. Stat. Phys. 14 (1963).

[6] Kugel, K. I., KHomsKII, D. I., JETP Lett. 15 (1972) 446 ; Sov. Phys. JETP 64 (1973) 1429, Solid State Commun. 13 (1973) 763.

[7] Cyrot, M., Lyon-Caen, C., J. Phys. C 6 (1973) L 247

[8] Weger, M., Phil. Mag. 24 (1971) 1095.

[9] Ashrenazi, J. \& Weger, M., Adv. Phys. 22 (1973) 207.

[10] INAGAKI, S. \& KUBO, R., Int. J. Magn. 4 (1973) 139.

[11] Yamada, H. \& Shimizu, M., J. Phys. Soc. Japan, 22 (1967) 1404.

[12] Goodenough, J. B., Mag. \& Chem. Bond (NY London) 1963.
[13] Kanomori, J., J. Appl. Phys. Suppl. 31 (1960) 145.

[14] Schneider, J., Heiner, E. \& HaUbenReisSer, W., Phys. Stat. Sol. (b) 53 (1972) 553.

[15] Capellmann, H., Phys. Rev. B 7 (1973) 4153.

[16] Kittel, C., Quantum Theory of Solids (J. Wiley \& Sons Inc.) 1966, p. 148.

[17] des Cloizeaux, J., J. Physique 20 (1959) 606.

[18] CoQblin, B. \& Blandin, A., Adv. Phys. 17 (1968) 281.

[19] des Cloizeaux, J., J. Physique 20 (1959) 751.

[20] Chao, K. A., Phys. Rev. B 8 (1973) 1088.

[21] Chang, K. Y., Young, W., J. Phys. C 4 (1971) 1855.

[22] Okazaki, A. \& Suemune, Y., J. Phys. Soc. Japan 16 (1961) 176.

[23] Jarrett, H. S., Cloud, W. H., Bouchard, R. J., Butler, S. R., Frederick, C. G. \& Gillson, J. L., Phys. Rev. Lett. 21 (1968) 617.

[24] Anderson, P. W., Phys. Rev. 115 (1959) 2. 